\title{
Computing the $p$-modulus of systems of measures via optimal plans
}

\author{
Mągorzata Ciska-Niedziałomska®
}

\begin{abstract}
We use the notion of the optimal plan associated with the Fuglede $p$-modulus of a family of Borel measures to derive formulas for the $p$-modulus and the extremal function in many special cases. Among others, we deduce Rodin's formula for a family of Hausdorff measures associated with leaves of a foliation defined by a single chart.
\end{abstract}

Mathematics Subject Classification. 28A25, 28A33.

Keywords. Fuglede $p$-modulus, Extremal function, Optimal plan, Rodin's formula.

1. Introduction. The notion of $p$-modulus was introduced by Fuglede [5] in order to study functional completion. Its reciprocal - extremal length, for $p=2$ - had been considered before and is closely related with the study of conformality and quasiconformality of maps on a plane.

Since the work of Fuglede, the $p$-modulus became a powerful tool in geometric measure theory. For example, Shanmugalingam [7] uses the $p$-modulus to consider $p$-harmonic maps on metric measure spaces. In such case, the $p$ modulus is considered for a family of curves, more precisely, for the family of 1-dimensional Hausdorff measures on continuous curves.

Computation of the $p$-modulus for a concrete family of measures is not easy and there are not many explicit formulas. The majority of them is related to a family of Hausdorff measures on curves or hypersurfaces and is related to the notion of $q$-capacity (here $p$ and $q$ are conjugate coefficients).

Recently, Ambrosio, di Marino, and Savare [1] characterised the $p$-modulus in terms of existence of a certain measure $\mathfrak{n}$ on the considered family of measures $\Sigma$ on $X$. This measure, called optimal plan, satisfies the following remarkable identity 


$$
\bmod _{p}(\Sigma)^{-1} \int_{X} f_{\Sigma}^{p-1}(x) f(x) d \mathfrak{m}=\int_{\Sigma} \int_{X} f(x) d \mu(x) d \mathfrak{n}(\mu), \quad f \geq 0,
$$

where $\bmod _{p}(\Sigma)$ is the $p$-modulus of $\Sigma$ (with respect to a fixed Borel measure $\mathfrak{m}$ ) and $f_{\Sigma}$ is an extremal function, i.e., a function which realizes the $p$-modulus. It can be shown that, up to a subfamily of modulus zero, $f_{\Sigma}$ is unique. Equality (1) allows us to derive formulas for the $p$-modulus and the extremal function in many special cases.

We begin with a general fact. We consider (1) without the assumption on extremality of $f_{\Sigma}$ and under two natural conditions we prove that $f_{\Sigma}$ is in fact extremal for the $p$-modulus of $\Sigma$ (Proposition 3).

The main part of this note is devoted to formulas for the $p$-modulus and the extremal function for different families of measures $\Sigma$. We heavily rely on (1) and Proposition 3. We rewrite (1) assuming there is a Borel bijection $\Sigma \mapsto Y$ for some Polish space $Y$ and we push forward the optimal plan $\mathfrak{n}$ to a probability measure on $Y$. In a sense, $Y$ counts elements of the family $\Sigma$ or, in other words, using geometrical language, is a transversal to $\Sigma$. We consider the following families:

1. measures which are absolutely continuous with respect to the reference measure $\mathfrak{m}$,

2. 'regular' family of measures with disjoint supports, in particular,

3. Lebesgue measures associated with leaves of a foliation defined by a single chart (Rodin's formula)

obtaining new, generalizing, or reproving well known relations.

2. Preliminary facts. Let $(X, d)$ be a Polish space and let $\mathfrak{m}$ be a (reference) Borel measure on $X$. Consider a family of Borel measures $\Sigma$. Let $\mathcal{L}(X, \mathfrak{m})$ (resp. $\left.\mathcal{L}_{+}^{p}(X, \mathfrak{m})\right)$ be the set of all (resp. nonnegative) $p$-integrable functions on $X$. We say that $f \in \mathcal{L}_{+}^{p}(X, \mathfrak{m})$ is admissible (for $\Sigma$ or, more precisely, for the $p$-modulus of $\Sigma$ ) if

$$
\int_{X} f(x) d \mu(x) \geq 1
$$

for all $\mu \in \Sigma$. Denote the family of such $f$ by $\operatorname{adm}_{p}(\Sigma)$. Then the $p$-modulus of $\Sigma$ is the following number

$$
\bmod _{p}(\Sigma)=\inf _{f \in \operatorname{adm}(\Sigma)} \int_{X} f^{p}(x) d \mathfrak{m}(x)
$$

if $\operatorname{adm}_{p}(\Sigma)$ is nonempty, otherwise we put $\bmod _{p}(\Sigma)=\infty$. An admissible function which realizes the $p$-modulus is called extremal. It can be shown, that up to a subfamily of full $p$-modulus, there is a unique extremal function [5]. Denote it by $f_{\Sigma}$.

We say that a certain condition $(P)$ holds for $p$-a.e. $\mu \in \Sigma$ if there is a subfamily $\Pi \subset \Sigma$ such that $(P)$ holds for every $\mu \in \Pi$ and $\bmod _{p}(\Sigma \backslash \Pi)=0$. The $p$-modulus has the following important properties [5]: 
(M1) $\bmod _{p}(\Sigma)=0$ if and only if there is an admissible function $f$ such that $\int_{X} f(x) d \mu(x)=\infty$ for every $\mu \in \Sigma$,

(M2) if $f \in \mathcal{L}^{p}(X, \mathfrak{m})$, then $f \in \mathcal{L}^{1}(X, \mu)$ for $p$-a.e. $\mu \in \Sigma$,

(M3) if $\Pi \subset \Sigma$, then $\bmod _{p}(\Pi) \leq \bmod _{p}(\Sigma)$,

(M4) if $\Pi \subset \bigcup_{i} \Sigma_{i}$, then $\bmod _{p}(\Pi) \leq \sum_{i} \bmod _{p}\left(\Sigma_{i}\right)$.

The $p$-modulus is a powerful tool, especially in the context of families of Hausdorff measures on curves, in geometric measure theory [7].

In the article [1], the authors prove the existence of an optimal plan associated with a family $\Sigma$. Let us be precise. Firstly, consider a space $\mathcal{M}(X)$ of positive and finite Borel measures on a Polish space $X$. We can equip this space with the weak-* topology and consider Borel probability measures on $\mathcal{M}(X)$. We say that such a measure $\mathfrak{n}$ is a plan with barycenter in $L^{q}(X, \mathfrak{m})$, where $q$ is a coefficient conjugate to $p$, if there is a constant $c(\mathfrak{n})$ such that

$$
\int_{\mathcal{M}(X)} \int_{X} f(x) d \mu(x) d \mathfrak{n}(\mu) \leq c(\mathfrak{n})\|f\|_{p}, \quad f \in \mathcal{L}_{+}(X, \mathfrak{m}) .
$$

The explanation of the use of $L^{q}(X, \mathfrak{m})$ is the following. Define a Borel measure $\underline{\mu}$ on $X$ as follows

$$
\underline{\mu}(A)=\int_{\Sigma} \mu(A) d \mathfrak{n}(\mu) .
$$

Then $\mu$ is absolutely continuous with respect to $\mathfrak{m}$ with a density $\rho$ belonging to $L^{q}(\bar{X}, \mathfrak{m})$. The authors in [1] prove the existence and remarkable properties of the optimal plan.

Theorem 1 ([1]). Assume $\Sigma \subset \mathcal{M}(X)$ is a Suslin set such that $\bmod _{p}(\Sigma)>0$ and $\sup _{\mu \in \Sigma} \mu(X)<\infty$. Then there is a plan $\mathfrak{n}$ with barycenter in $L^{q}(X, \mathfrak{m})$ such that $\mathfrak{n}$ is concentrated on $\Sigma, c(\mathfrak{n})=\bmod _{p}(\Sigma)^{-\frac{1}{p}}$ is the best possible constant, and the following formulas hold

$$
\int_{X} f_{\Sigma}(x) d \mu(x)=1 \quad \text { for } \mathfrak{n}-\text { a.e. } \mu \quad \text { and } \quad \rho=\frac{f_{\Sigma}^{p-1}}{\left\|f_{\Sigma}\right\|_{p}^{p}},
$$

where $f_{\Sigma}$ is the extremal function for $\Sigma$.

Remark 2. Let us recall (see [3]) that a Polish space is Suslin and the Borel image of a Suslin set is again Suslin.

By the definition of $\rho$, we immediately see that a formula for $\rho$ in the above Theorem implies

$$
\int_{\Sigma} \int_{X} f(x) d \mu(x) d \mathfrak{n}(\mu)=\bmod _{p}(\Sigma)^{-1} \int_{X} f_{\Sigma}^{p-1}(x) f(x) d \mathfrak{m}(x)
$$

for any nonnegative Borel function $f$. 
2.1. An important consequence. Let us prove a general consequence of the formula (3).

Now, we give one implication of the formula (3) but without the assumption on extremality of $f_{\Sigma}$. This approach has been partially noticed in the paper [1] but in the context with relation (2) and with different conclusions.

Assume there is a probability measure $\eta$ on $\mathcal{M}(X)$ such that for any nonnegative Borel $f$,

$$
\int_{\mathcal{M}(X)} \int_{X} f(x) d \mu(x) d \eta(\mu)=\int_{X} f_{\eta}^{p-1}(x) f(x) d \mathfrak{m}(x)
$$

for some function $f_{\eta} \in \mathcal{L}_{+}^{p}(X, \mathfrak{m})$. Then, by the Hölder inequality,

$$
\int_{\mathcal{M}(X)} \int_{X} f(x) d \mu(x) d \eta(\mu) \leq\left\|f_{\eta}^{p-1}\right\|_{q} \cdot\|f\|_{p}
$$

hence $\eta$ is a plan with barycenter in $L^{q}(X, \mathfrak{m})$ with $c(\eta)=\left\|f_{\eta}^{p-1}\right\|_{q}$. Moreover, if $f \in \mathcal{L}_{+}^{p}(X, \mathfrak{m})$ is admissible, then

$$
\eta(\Sigma) \leq\left\|f_{\eta}^{p-1}\right\|_{q} \cdot\|f\|_{p} .
$$

Hence, taking the infimum with respect to all admissible functions $f$, we get

$$
\frac{\eta(\Sigma)^{p}}{\left\|f_{\eta}^{p-1}\right\|_{q}^{p}} \leq \bmod _{p}(\Sigma)=\frac{1}{c(\mathfrak{n})}
$$

Consider the following two assumptions:

(E1) the measure $\eta$ is concentrated on $\Sigma$,

(E2) there is a constant $c>0$ such that for every $\mu \in \Sigma$,

$$
\int_{X} f_{\eta}(x) d \mu(x)=c .
$$

Proposition 3. Assume $\Sigma \subset \mathcal{M}(X)$ is a Suslin set such that $\bmod _{p}(\Sigma)>0$ and $\sup _{\mu \in \Sigma} \mu(X)<\infty$. Assume, moreover, that there is a probability measure $\eta$ on $\mathcal{M}(X)$ such that (4) holds for some $f_{\eta} \in \mathcal{L}_{+}^{p}(X)$. If (E1), (E2) hold, then

(i) $c=\bmod _{p}(\Sigma)^{1-q}$,

(ii) $f_{\Sigma}=\bmod _{p}(\Sigma)^{q-1} f_{\eta}$ is extremal for the p-modulus of $\Sigma$,

(iii) $\eta=\mathfrak{n}$, i.e., $\eta$ is the optimal plan with barycenter in $L^{q}(X, \mathfrak{m})$,

(iv) (4) is equivalent to (3).

Proof. Substituting $f=f_{\eta}$ in (4), we have

$$
c=\int_{X} f_{\eta}^{p}(x) d \mathfrak{m}(x) .
$$

Thus, by (3) with $f=f_{\eta}$, we have

$$
c=\bmod _{p}(\Sigma)^{-1} \int_{X} f_{\Sigma}^{p-1}(x) f_{\eta}(x) d \mathfrak{m}(x) \leq \bmod _{p}(\Sigma)^{-\frac{1}{p}} c^{\frac{1}{p}} .
$$


Hence

$$
c^{\frac{1}{q}} \bmod _{p}(\Sigma)^{\frac{1}{p}} \leq 1
$$

On the other hand, $f_{\Sigma}$ is admissible for the $p$-modulus, so by (5),

$$
1 \leq c^{\frac{1}{q}} \bmod _{p}(\Sigma)^{\frac{1}{p}}
$$

Together with the previous inequality, we conclude (i). For the proof of (ii), notice that $c^{-1} f_{\eta}$ is admissible for the $p$-modulus of $\Sigma$ and

$$
\bmod _{p}(\Sigma)=c^{1-p}=c^{-p} \int_{X} f_{\eta}^{p}(x) d \mathfrak{m}(x)=\int_{X}\left(c^{-1} f_{\eta}\right)^{p}(x) d \mathfrak{m}(x),
$$

which proves the extremality of $c^{-1} f_{\eta}$. By the uniqueness (up to a subset of zero measure), we get (ii). The remaining conditions follow immediately by (i) and (ii).

3. Results. Adopt notation from the previous section. Fix $p>1$ and let $\Sigma$ be a family of Borel measures such that $\bmod _{p}(\Sigma)>0$ and $\sup _{\mu \in \Sigma} \mu(X)<\infty$. Assume that the family $\Sigma \subset \mathcal{M}(X)$ can be realized as follows: there is a Borel bijective map $\mu: Y \rightarrow \Sigma$ with a Borel inverse map $\mu^{-1}: \Sigma \rightarrow Y$, where $Y$ is a Polish space. Hence, we may index the family $\Sigma$ with elements from $Y$, i.e., $\Sigma=\left\{\mu_{y}\right\}_{y \in Y}$. We may push-forward the optimal plan $\mathfrak{n}$ with respect to $\mu^{-1}$ to obtain a Borel probability measure $\lambda=\lambda_{\mathfrak{n}}$ on $Y$. Then formula (3) may be rewritten as follows

$$
\int_{Y} \int_{X} f(x) d \mu_{y}(x) d \lambda(y)=\bmod _{p}(\Sigma)^{-1} \int_{X} f_{\Sigma}^{p-1}(x) f(x) d \mathfrak{m}
$$

for any nonnegative Borel function $f$.

3.1. Measures absolutely continuous with respect to $\mathfrak{m}$. In this section, we provide a formula for the $p$-modulus in the case when each $\mu_{y} \in \Sigma$ is absolutely continuous with respect to $\mathfrak{m}$.

Proposition 4. Assume there is a Borel function $\alpha=\alpha(x, y): X \times Y \rightarrow \mathbb{R}_{+}$ such that $\mu_{y}=\alpha(\cdot, y) \mathfrak{m}$. Put

$$
\hat{\alpha}(x)=\int_{Y} \alpha(x, y) d \lambda(y)
$$

Then

$$
f_{\Sigma}(x)=\frac{\hat{\alpha}^{q-1}(x)}{\int_{X} \hat{\alpha}^{q}(x) d \mathfrak{m}(x)} \quad \text { and } \quad \bmod _{p}(\Sigma)=\left(\int_{X} \hat{\alpha}^{q}(x) d \mathfrak{m}(x)\right)^{1-p} .
$$

Proof. In this case, formula (7) simplifies to

$$
\int_{Y} \int_{X} \alpha(x, y) f(x) d \mathfrak{m} d \lambda(y)=\bmod _{p}(\Sigma)^{-1} \int_{X} f_{\Sigma}^{p-1}(x) f(x) d \mathfrak{m} .
$$


Since $f \geq 0$ is arbitrary, by the Fubini-Tonelli theorem, we get

$$
\int_{Y} \alpha(x, y) d \lambda(y)=\bmod _{p}(\Sigma)^{-1} f_{\Sigma}^{p-1}(x), \quad x \in X .
$$

Hence $f_{\Sigma}(x)=\left(\bmod _{p}(\Sigma) \hat{\alpha}(x)\right)^{q-1}$. Thus

$$
\bmod _{p}(\Sigma)=\bmod _{p}(\Sigma)^{q} \int_{X} \hat{\alpha}^{q}(x) d \mathfrak{m},
$$

which implies the formula for the $p$-modulus and for the extremal function.

If $\alpha$ has separate variables, the formulas are much simpler and, what is probably the most important, we get some additional information about the measure $\lambda$.

Corollary 5. Assume $\alpha$ is of the form $\alpha(x, y)=\beta(x) \gamma(y)$. Then

$$
f_{\Sigma}(x)=\frac{\beta^{q-1}(x)}{\left(\int_{Y} \gamma(y) d \lambda(y)\right)^{q-1} \int_{X} \beta^{q}(x) d \mathfrak{m}(x)}
$$

and

$$
\bmod _{p}(\Sigma)=\left(\int_{Y} \gamma(y) d \lambda(y)\right)^{-p}\left(\int_{X} \beta^{q}(x) d \mathfrak{m}\right)^{1-p} .
$$

Proof. It suffices to notice that

$$
\hat{\alpha}(x)=\left(\int_{Y} \gamma(y) d \lambda(y)\right) \beta(x),
$$

and use Proposition 4.

Corollary 6. If $\alpha(x, y)=\beta(x) \gamma(y)$, then $\gamma$ is constant on a subset of $Y$ of full $\lambda$-measure.

Proof. By Theorem 1, we know that $\int_{X} f_{\Sigma}(x) d \mu_{y_{0}}(x)=1$ for $\lambda$-a.e. $y_{0} \in Y$. Thus, for $\lambda$-a.e. $y_{0} \in Y$,

$$
1=\frac{\int_{X} \beta^{q-1}(x) \beta(x) \gamma\left(y_{0}\right) d \mathfrak{m}}{\left(\int_{Y} \gamma(y) d \lambda(y)\right)^{q-1} \int_{X} \beta^{q}(x) d \mathfrak{m}(x)}=\frac{\gamma\left(y_{0}\right)}{\int_{Y} \gamma(y) d \lambda(y)} .
$$

Denoting the denominator on the right hand side of the above formula by $c$, we have $\gamma\left(y_{0}\right)=c$ for $\lambda$-a.e. $y_{0} \in Y$.

3.2. 'Regular' family of measures with disjoint supports. Here, we consider a special case of a separate system of measures $\Sigma$.

Proposition 7. Assume there is a Borel map $\alpha: L \times Y \rightarrow X$ and Borel functions $\beta, \gamma: L \times Y \rightarrow \mathbb{R}_{+}$, where $(L, \nu)$ is a Polish space with a Borel measure $\nu$, such that

$$
\alpha(\cdot, y)_{\sharp}(\beta(\cdot, y) \nu)=\mu_{y}, \quad \alpha_{\sharp}(\gamma(\nu \otimes \lambda))=\mathfrak{m} .
$$


Then, for $\lambda$-a.e. $y_{0}$,

$$
f_{\Sigma}(\alpha(z, y))=\left(\int_{L} \frac{\beta^{q}\left(z, y_{0}\right)}{\gamma^{q-1}\left(z, y_{0}\right)} d \nu(z)\right)^{-1} \frac{\beta^{q-1}(z, y)}{\gamma^{q-1}(z, y)}
$$

and

$$
\bmod _{p}(\Sigma)=\left(\int_{L} \frac{\beta^{q}\left(z, y_{0}\right)}{\gamma^{q-1}\left(z, y_{0}\right)} d \nu(z)\right)^{1-p}
$$

Proof. By the assumptions, formula (7) simplifies to (for any nonnegative Borel $\operatorname{map} f$ )

$$
\begin{aligned}
& \int_{L \times Y} f(\alpha(z, y)) \beta(z, y) d \nu(z) d \lambda(y) \\
& \quad=\bmod _{p}(\Sigma)^{-1} \int_{L \times Y} f_{\Sigma}^{p-1}(\alpha(z, y)) f(\alpha(z, y)) \gamma(z, y) d \nu(z) d \lambda(y) .
\end{aligned}
$$

Hence

$$
\beta(z, y)=\bmod _{p}(\Sigma)^{-1} f_{\Sigma}^{p-1}(\alpha(z, y)) \gamma(z, y)
$$

Therefore

$$
f_{\Sigma}(\alpha(z, y))=\bmod _{p}(\Sigma)^{q-1}\left(\frac{\beta(z, y)}{\gamma(z, y)}\right)^{q-1} .
$$

By Theorem 1, for $\lambda$-a.e. $y$, we have

$$
\bmod _{p}(\Sigma)=\left(\int_{L} \frac{\beta^{q}(z, y)}{\gamma^{q-1}(z, y)} d \nu(z)\right)^{1-p} .
$$

This also implies the formula for $f_{\Sigma}$.

Remark 8. Notice that the function of the variable $y$ on the right hand side of (9) is constant on a set of full $\lambda$-measure in $Y$.

Let us prove, in a sense, the converse statement by using Proposition 3.

Proposition 9. Assume there is a Borel bijective map $\alpha: L \times Y \rightarrow X$ and Borel positive functions $\beta, \gamma: L \times Y \rightarrow \mathbb{R}$, where $(L, \nu)$ and $(Y, \eta)$ are Polish spaces with a Borel measure $\nu$ and a Borel probability measure $\eta$, such that

$$
\alpha(\cdot, y)_{\sharp}(\beta(\cdot, y) \nu)=\mu_{y}, \quad \alpha_{\sharp}(\gamma(\nu \otimes \eta))=\mathfrak{m} .
$$

If, additionally, $\frac{\beta}{\gamma} \circ \alpha^{-1} \in \mathcal{L}^{q}(X, \mathfrak{m})$ and the function

$$
y \mapsto \int_{L} \frac{\beta^{q}(z, y)}{\gamma^{q-1}(z, y)} d \nu(z)
$$

is constant, then the extremal function $f_{\Sigma}$ for the p-modulus of $\Sigma$ and the $p$-modulus $\bmod _{p}(\Sigma)$ are given by (8) and (9) (for any $\left.y_{0}\right)$. 
Proof. By assumptions and the Fubini-Tonelli theorem, we have

$$
\begin{aligned}
\int_{Y} \int_{X} f(x) d \mu_{y}(x) d \eta(y) & =\int_{L} \int_{Y} f(\alpha(z, y)) \beta(z, y) d \eta(y) d \nu(z) \\
& =\int_{X} \frac{\beta}{\gamma} \circ \alpha^{-1}(x) f(x) d \mathfrak{m}(x)
\end{aligned}
$$

for any Borel function $f \geq 0$. Thus (4) holds with $f_{\eta}=\left(\frac{\beta}{\gamma} \circ \alpha^{-1}\right)^{q-1}$. Moreover, for any $y \in Y$,

$$
\int_{X} f_{\eta}(x) d \mu_{y}(x)=\int_{L} \frac{\beta^{q}(z, y)}{\gamma^{q-1}(z, y)} d \nu(z) .
$$

Hence, we may apply Proposition 3.

Example 10 (Rodin's formula). The original Rodin formula [6] is the formula for the extremal function for the $p$-modulus of a family of curves on the Euclidean plane, which are images of horizontal lines in the rectangle $[0,1] \times[0, b]$. It was generalized to arbitrary dimension [2] (still for a family of curves) and recently, by the author and K. Niedziałomski, to any family of $k$-dimensional surfaces defined by a single chart [4]. Let us recall this result and show how it can be obtained from Proposition 9.

Theorem 11 ([4]). Let $L$ and $Y$ be two domains in $\mathbb{R}^{m}$ and $\mathbb{R}^{n-m}$, respectively. Let $\alpha: L \times Y \rightarrow \Omega$ be a $C^{1}$-smooth diffeomorphism onto $\Omega \subset \mathbb{R}^{n}$. Denote by $\Sigma$ the family of $m$-dimensional surfaces $\sigma_{y}, y \in Y$, being the images of $L$ with respect to $f, \sigma_{y}=\alpha(L, y)$. Then the extremal function for the p-modulus of $\Sigma$ is the following

$$
f_{\Sigma}(x)=\frac{1}{l(z)}\left(\frac{\left|J_{\alpha}^{z}\right|}{\left|J_{\alpha}\right|}\right)^{q-1} \circ \alpha^{-1}(x), \quad x=\alpha(z, y),
$$

where

$$
l(y)=\int_{L}\left(\frac{\left|J_{\alpha}^{z}\right|}{\left|J_{\alpha}\right|}\right)^{q} J_{\alpha} d z .
$$

Moreover, the p-modulus of $\Sigma$ equals

$$
\bmod _{p}(\Sigma)=\int_{Y} l(y)^{1-p} d y .
$$

Recall that $J_{\alpha}^{z}$ is the Jacobian of a map $z \mapsto \alpha(z, y)$ with fixed $z$, whereas $J_{\alpha}$ is the 'full' Jacobian of $\alpha$.

Let $\nu=d z$ be the Lebesgue measure on $L$ and $\mathfrak{m}=d x$ be the Lebesgue measure on $\Omega$ and let $\mu_{y}$ be the Lebesgue measure on $\sigma_{y}$. Let us define $\beta$ and $\gamma$ by

$$
\beta(z, y)=\left|J^{z} \alpha(z, y)\right|, \quad \gamma(z, y)=\frac{\left|J_{\alpha}(z, y)\right|}{\rho(y)}
$$


where $\rho(y)=c l(y)^{1-p}$ and $c$ is a constant such that the measure

$$
\eta=\rho d y
$$

is a probability measure on $Y$, i.e., $c^{-1}=\int_{Y} l(y)^{1-p} d y$. Since, by the FubiniTonelli theorem, for any nonnegative Borel $f$,

$$
\begin{aligned}
\int_{Y} \int_{L} f(\alpha(z, y)) \gamma(z, y) d \nu(z) d \eta(y) & =\int_{Y} \int_{L} f(\alpha(z, y))\left|J_{\alpha}\right| d z d y \\
& =\int_{X} f(x) d \mathfrak{m}(x),
\end{aligned}
$$

we have $\alpha_{\sharp}(\gamma \nu \otimes \eta)=\mathfrak{m}$. Clearly, $\alpha(\cdot, y)_{\sharp}(\beta(\cdot, y) \nu)=\mu_{y}$. Moreover,

$$
\int_{L} \frac{\beta^{q}(z, y)}{\gamma^{q-1}(z, y)} d \nu(z)=\int_{L} c^{q-1} \frac{\left|J_{\alpha}^{z}(z, y)\right|^{q}}{\left|J_{\alpha}(z, y)\right|^{q-1}} l(y)^{-1} d z=c^{q-1}
$$

for any $y \in Y$. Thus the assumptions of Proposition 9 are satisfied. Hence (12) and (11) hold.

Open Access. This article is licensed under a Creative Commons Attribution 4.0 International License, which permits use, sharing, adaptation, distribution and reproduction in any medium or format, as long as you give appropriate credit to the original author(s) and the source, provide a link to the Creative Commons licence, and indicate if changes were made. The images or other third party material in this article are included in the article's Creative Commons licence, unless indicated otherwise in a credit line to the material. If material is not included in the article's Creative Commons licence and your intended use is not permitted by statutory regulation or exceeds the permitted use, you will need to obtain permission directly from the copyright holder. To view a copy of this licence, visit http://creativecommons. org/licenses/by/4.0/.

Publisher's Note Springer Nature remains neutral with regard to jurisdictional claims in published maps and institutional affiliations.

\section{References}

[1] Ambrosio, L., Di Marino, S., Savare, G.: On the duality between p-modulus and probability measures. J. Eur. Math. Soc. 17(8), 1817-1853 (2015)

[2] Brakalova, M., Markina, I., Vasil'ev, A.: Extremal functions for modules of systems of measures. J. Anal. Math. 133, 335-359 (2017)

[3] Bogachev, V.: Measure Theory, vols. I and II. Springer, Berlin (2007)

[4] Ciska-Niedziałomska, M., Niedziałomski, K.: Rodin's formula in arbitrary codimension. Ann. Acad. Sci. Fenn. Math. 44(1), 221-229 (2019)

[5] Fuglede, B.: Extremal length and functional completion. Acta Math. 98, 171-219 (1957)

[6] Rodin, B.: The method of extremal length. Bull. Am. Math. Soc. 80, 587-606 (1974) 
[7] Shanmugalingam, N.: Harmonic functions on metric spaces. Illinois J. Math. 45(3), 1021-1050 (2001)

\section{Mągorzata Ciska-Niedziąomska}

Department of Mathematics and Computer Science

University of Łódź

ul. Banacha 22

90-238 Lodz

Poland

e-mail: malgorzata.niedzialomska@wmii.uni.lodz.pl

Received: 22 January 2020 